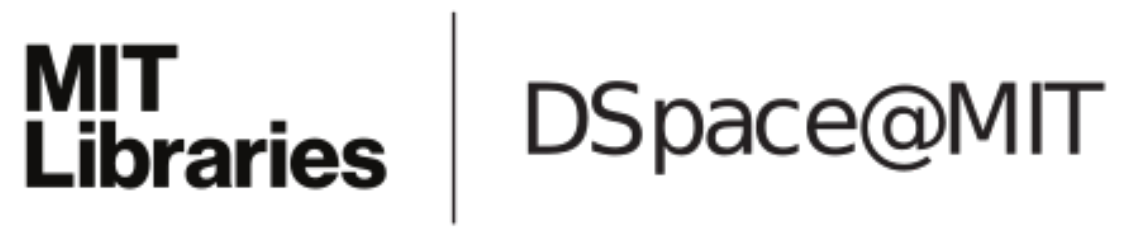

\author{
MIT Open Access Articles
}

Atom-Based Bootstrap Embedding For Molecules

The MIT Faculty has made this article openly available. Please share how this access benefits you. Your story matters.

Citation: Ye, Hongzhou and Troy Van Voorhis. "Atom-Based Bootstrap Embedding For Molecules." Journal of Physical Chemistry Letters 10, 20 (October 2019): 6368-6374 ( 2019 American Chemical Society

As Published: https://pubs.acs.org/doi/10.1021/acs.jpclett.9b02479

Publisher: American Chemical Society (ACS)

Persistent URL: https://hdl.handle.net/1721.1/128160

Version: Author's final manuscript: final author's manuscript post peer review, without publisher's formatting or copy editing

Terms of Use: Article is made available in accordance with the publisher's policy and may be subject to US copyright law. Please refer to the publisher's site for terms of use. 


\title{
Atom-Based Bootstrap Embedding For Molecules
}

\author{
Hong-Zhou Ye and Troy Van Voorhis* \\ Department of Chemistry, Massachusetts Institute of Technology, Cambridge, MA 02139 \\ E-mail: tvan@mit.edu
}

Abstract. Recent developments in quantum embedding have offered an attractive approach to describing electron correlation in molecules. However, previous methods such as Density Matrix Embedding Theory (DMET) require rigid partitioning of the system into fragments, which creates significant ambiguity for molecules. Bootstrap Embedding (BE) is more flexible because it allows overlapping fragments, but when done on an orbital-byorbital basis BE introduces ambiguity in defining the connectivity of the orbitals. In this Letter, we present an atom-based fragment definition that significantly augments BE's performance in molecules. The resulting method, which we term atom-based BE, is very effective at recovering valence electron correlation in moderate-sized bases and delivers nearchemical-accuracy results using extrapolation. We anticipate atom-based BE may lead to a low-scaling and highly accurate approach to electron correlation in large molecules.

\section{TOC Graphic}

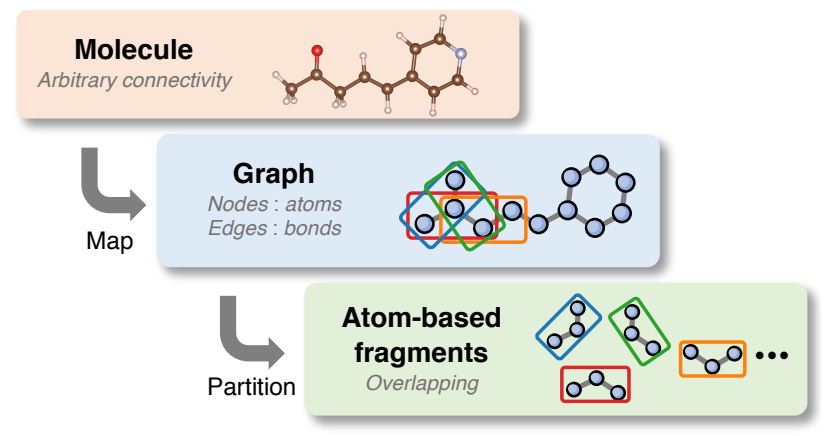

In quantum chemistry, an accurate description of electron correlation is crucial to achieve chemical accuracy $(<1 \mathrm{kcal} / \mathrm{mol})$. From first principles, achieving this level of accuracy requires correlated wave function ${ }^{1-4}$ (CWF) calculations whose computational cost grows supralinearly with system size $\left[O\left(N^{p}\right)\right.$ with $p \geq$ 5 typically]. Kohn-Sham density functional theory $^{5,6}$ (KS-DFT) provides a relatively modestcost $\left[O\left(N^{3}\right)\right]$ solution to the problem and has achieved success in weakly correlated systems. However, the lack of systematic improvability in the approximate exchange-correlation functionals has limited its usage in more challenging scenarios. $^{7-9}$

Recent developments in quantum embedding have offered an attractive solution to this problem. ${ }^{10-19}$ These methods bypass the scaling curse of the CWF methods by applying them only to smaller and hence manageable fragments of a large system, while leaving the interaction between the fragments and the rest of the system described in a lower level of theory (i.e., bath). In this work, we focus on a specific class of quantum embedding methods based on the Schmidt decomposition ${ }^{20,21}$ (SD), which preserves the entanglement between fragments and baths and hence is especially suitable for embedding chemical systems. For instance, Density Matrix Embedding Theory ${ }^{13,14}$ (DMET) has demonstrated success in treating electron correlation in lattice models ${ }^{13,22,23}$ and simple chemical systems. ${ }^{14,24-27}$

Similar to other embedding methods, ${ }^{28,29}$ SDbased embedding with non-overlapping fragments tends to converge slowly towards the exact solution with increasing fragment size. 
This is due to the persistent surface error in the description of the interactions between the fragment edges and their respective baths. To tackle this problem, we developed Bootstrap Embedding ${ }^{15}$ (BE) that uses overlapping fragments to directly improve the description of fragment edges. BE shows an exponential rate of convergence with fragment size in lattice models, which is much faster than the decay of surface error in DMET. ${ }^{15}$ However, recent attempts of generalizing BE to molecules have proved challenging, primarily due to the lack of a good partition scheme for molecules. ${ }^{16,30,31}$

In this Letter, we present a better fragment definition that enables black-box partition of molecules into overlapping fragments based on atoms and chemical bonds. This atom-based partition scheme significantly augments BE's performance, leading to a fast convergent embedding method for the valence correlation (i.e., the correlation energy one would obtain by performing a full-valence complete active-space calculation) of molecules.

We start by briefly summarizing the fundamental aspects of SD-based embedding methods (see Ref. 25 for a detailed review). Suppose the molecule is described by a Hamiltonian of $N$ atom-centered orbitals. In second quantization:

$$
\hat{H}=\sum_{\mu \nu}^{N} h_{\mu \nu} c_{\mu}^{\dagger} c_{\nu}+\frac{1}{2} \sum_{\mu \nu \lambda \sigma}^{N} V_{\mu \nu \lambda \sigma} c_{\mu}^{\dagger} c_{\lambda}^{\dagger} c_{\sigma} c_{\nu} .
$$

Given any choice of $N_{f}$ orbitals that compose our fragment (which we assume to be a minority), the exact eigenstate of $\hat{H}$ can be written in a tensor-product form via the Schmidt Decomposition (SD)

$$
|\Psi\rangle=\sum_{i}^{N_{f}} \lambda_{i}\left|\tilde{f}_{i}\right\rangle\left|\tilde{b}_{i}\right\rangle,
$$

where $\left\{\left|\tilde{f}_{i}\right\rangle\right\}$ and $\left\{\left|\tilde{b}_{i}\right\rangle\right\}$ are the many-body fragment and bath states, and $\lambda_{i} \in[0,1]$ characterizes the entanglement between $\left|\tilde{f}_{i}\right\rangle$ and $\left|\tilde{b}_{i}\right\rangle$. Eqn (2) leads to an exact embedding since $|\Psi\rangle$ is also an eigenstate of the projected Hamilto- nian, $\hat{H}_{\mathrm{emb}}=\hat{P}_{f b} \hat{H} \hat{P}_{f b}$, where

$$
\hat{P}_{f b}=\sum_{i, j}^{N_{f}}\left|\tilde{f}_{i}\right\rangle\left|\tilde{b}_{j}\right\rangle\left\langle\tilde{f}_{i}\right|\left\langle\tilde{b}_{j}\right| .
$$

However, this procedure has little practical use because it requires the exact wave function to begin with.

SD-based embedding methods adopt an approximate bath wave function - typically a mean-field wave function, with only few exceptions. ${ }^{32,33}$ In a mean-field SD, the complicated many-body bath states $\left\{\left|\tilde{b}_{i}\right\rangle\right\}$ become two sets of orbitals - the entangled bath $\left\{\left|b_{i}\right\rangle\right\}$ and the pure environment $\left\{\left|e_{i}\right\rangle\right\}$ - depending on whether they are entangled with the particular set of fragment orbitals $\left\{\left|f_{i}\right\rangle\right\}$. Assuming no linear dependence issues, for a fragment of size $N_{f}$, there are $N_{b}=N_{f}$ entangled bath orbitals. These $2 N_{f}$ orbitals form an active space with the remaining environment orbitals being spectators, giving rise to the following embedding Hamiltonian,

$$
\hat{H}_{\mathrm{emb}}=\sum_{i j}^{N_{f}+N_{b}} \bar{h}_{i j} a_{i}^{\dagger} a_{j}+\frac{1}{2} \sum_{i j k l}^{N_{f}+N_{b}} \bar{V}_{i j k l} a_{i}^{\dagger} a_{k}^{\dagger} a_{l} a_{j},
$$

where

$$
\begin{aligned}
\bar{h}_{i j} & =\sum_{\mu \nu}^{N} T_{\mu i}^{f b} F_{\mu \nu}^{e} T_{\nu j}^{f b}, \\
\bar{V}_{i j k l} & =\sum_{\mu \nu \lambda \sigma}^{N} T_{\mu i}^{f b} T_{\nu j}^{f b} V_{\mu \nu \lambda \sigma} T_{\lambda k}^{f b} T_{\sigma l}^{f b},
\end{aligned}
$$

where $\mathbf{T}^{f b}$ is the coefficient matrix of $\left\{\left|f_{i}\right\rangle\right\}$ and $\left\{\left|b_{i}\right\rangle\right\}$, and $\mathbf{F}^{e}$ is the environment Fock matrix. Note that $\hat{H}_{\text {emb }}$ is exactly a two particle operator. This feature is unique to mean-field baths and motivates the choice of these wave functions for practical embedding.

For reasonably sized fragments, $\hat{H}_{\mathrm{emb}}$ can be solved using accurate CWF methods to recover the electron correlation missing in the approximate bath. The main difference between DMET (and its variants) and BE lies in the matching conditions employed to optimize the embedding. DMET, often used with 
(a)

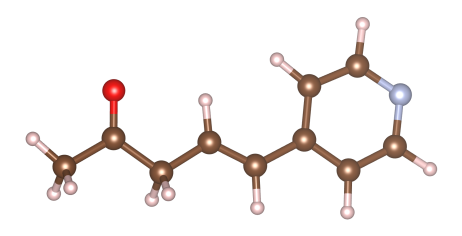

Graph

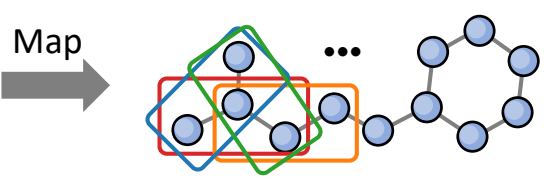

Atom-based

fragments (b)

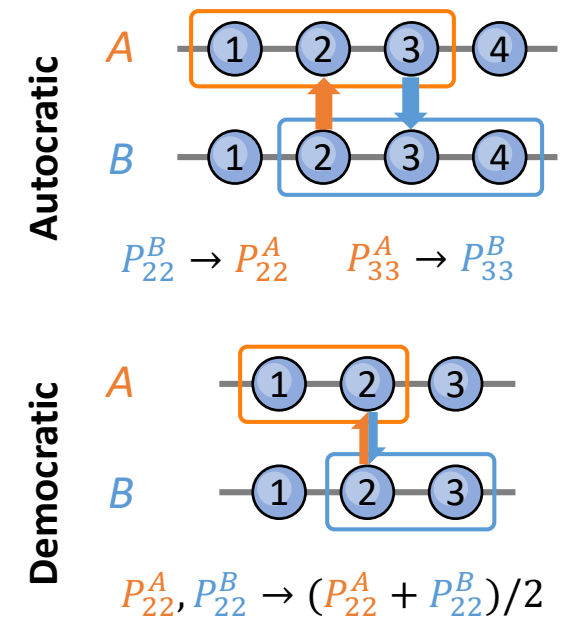

(c)

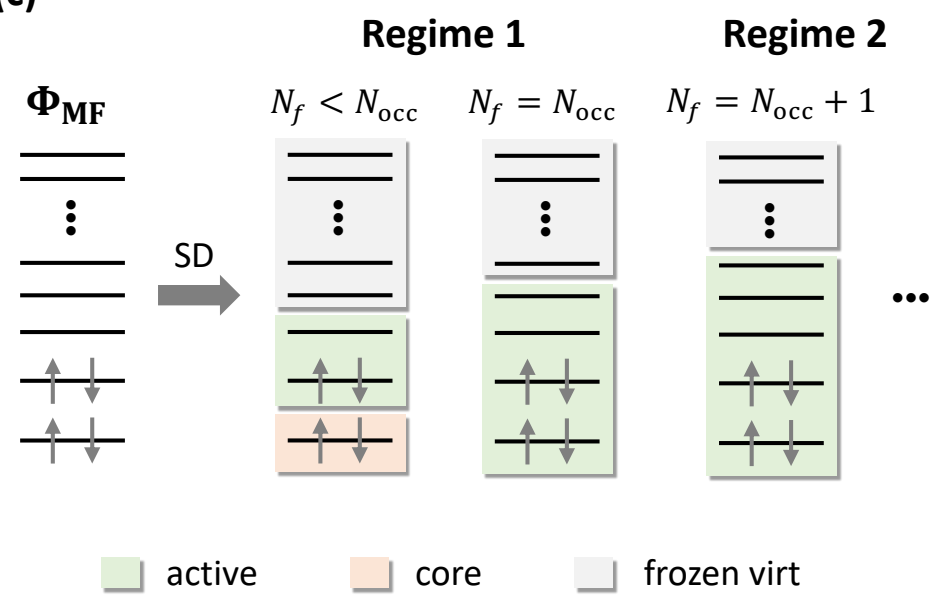

Figure 1: (a) Schematic illustration of the atom-based partition scheme for generating overlapping fragments. (b) Two different types of matching conditions arising from using overlapping fragments. (c) Schematic illustration of two distinct regimes of Schmidt decomposing a mean-field wave function, $\Phi_{\mathrm{MF}}$. For $N_{f} \leq N_{\mathrm{occ}}$, adding a new fragment orbital adds two orbitals (one occupied and one virtual) to the active space (regime 1). Once $N_{f}>N_{\text {occ }}$, however, adding a new fragment orbital only adds one virtual orbital to the active space (regime 2).

non-overlapping fragments, matches the density matrix of the mean field to that of the CWF calculation for each fragment. ${ }^{13,14,27,34,35}$ $\mathrm{BE}$, on the other hand, uses overlapping fragments and matches the density matrix of one fragment with another in their overlapping region. ${ }^{15,30,31}$

The most natural way to translate $\mathrm{BE}$ to molecules is to use atoms, rather than individual orbitals or lattice sites (as done in previous work ${ }^{31}$ ), as the fundamental building block. That is, in an atom-centered basis it is most natural that we view all the basis functions on an atom as a group when constructing fragments. This idea is illustrated in Figure 1a. We first map a molecule onto a graph whose nodes and edges correspond to atoms (hydrogens are grouped with the heavy atoms they are bonded to) and chemical bonds of that molecule. Then, we form fragments by finding all connected sub- graphs of a given size (say $n$ atoms). A specific example is provided in Figure S10. Fragments generated in this way overlap in many different topologies, giving rise to two types of matching conditions as shown in Figure 1b.

In autocratic matching, the overlapping region (e.g., atom 2) is more accurately described in fragment $A$ than in fragment $B$. We thus can improve the embedding of fragment $B$ by constraining its density matrix elements on atom 2 to match those calculated from using fragment $A$ (orange arrow in Figure 1b). A similar constraint can be placed on atom 3 with an opposite direction (blue arrow in Figure 1b). In democratic matching, the overlapping region is described equally (well or poorly) in both fragments. In this case, we make a democratic choice and require the density matrix elements computed using both fragments match their arithmetic mean in the overlapping region. 
These prototypes of matching conditions can be extended to more general cases as detailed in Sec. S3. As a result, this atom-based fragment definition offers a black-box approach to applying BE to molecules whenever a clear definition of interatomic connectivity (i.e., chemical bonds) exists.

We note that the idea of using overlapping fragments to accelerate the convergence of embedding has also been explored in different contexts. ${ }^{18,36,37}$ However, to the best of our knowledge, none of these methods exploit the matching conditions described above to further improve the embedding. As we will see in numerical examples, the effect of density matching can be significant for SD-based embedding for molecules.

Before presenting results, we briefly discuss the convergence of $\mathrm{BE}$ with fragment size. Ideally, an embedding method should converge fast and monotonically to the full-system result by enlarging the fragments. Furthermore, if the error decays by a known functional form, extrapolation based on small-fragment calculations would serve the purpose efficiently. Due to the nature of SD, BE is exact when the fragment and entangled bath together span the full Hilbert space. However, the loss of entanglement in the low-level, mean-field wave function can hinder extrapolation.

Typically, in mean-field SD, each fragment orbital $\left|f_{i}\right\rangle$ produces exactly one entangled bath orbital $\left|b_{i}\right\rangle \cdot{ }^{14,22}$ This pair of orbitals spans exactly one occupied and one virtual orbital. However, the behavior changes abruptly as the number of fragment orbitals $\left(N_{f}\right)$ increases (Figure 1c). When $N_{f}$ is less than the number of occupied orbitals $\left(N_{\text {occ }}\right)$, every new fragment orbital contributes two orbitals to the active space. Once $N_{f} \geq N_{\text {occ }}$, however, all of the occupied orbitals will already be present in the active space. Thus, adding a new fragment orbital only adds a single virtual orbital to the active space. We note that this problem is not very serious in lattice models, where the number of virtual orbitals $\left(N_{\text {virt }}\right)$ is typically the same as or similar to the number of occupieds, making the embedding stay in regime 1 for all reasonable fragment sizes. But for molecular calculations - where $N_{\text {virt }}$ typically greatly exceeds $N_{\text {occ }}$ - this discontinuous behavior is expected to be more problematic, as we shall see below.

In order to demonstrate the utility of this atom-based embedding strategy, we implemented it for the simple case of MP2 (MøllerPlesset second-order perturbation theory ${ }^{1}$ )-inRHF (spin-restricted Hartree-Fock) embedding and tested it for some prototypical molecular systems. This choice of an inexpensive local solver allows us to treat very large fragments, and helps characterize the convergence behavior of atom-based BE with fragment size. We note that MP2 is known to be insufficient for strong electron correlation, ${ }^{38,39}$ and so our results are not expected to be chemically accurate. Instead, we will benchmark the fragment calculations against the full-system MP2 results and focus on precision.

Atom-centered Gaussian orbitals localized using the Foster-Boys scheme ${ }^{40}$ are used as the computational basis (generated in QChem 5.0 $\left.{ }^{41}\right)$. All embedding calculations are performed in frankenstein, ${ }^{42}$ which uses Libint $2^{43}$ for atomic integrals evaluation and $\mathrm{PySCF}^{44}$ for the MP2 solver. Comparison between BE and DMET is made whenever possible. The RHF orbitals are not optimized in either method (we hence use the acronym DMET0 below), but in BE, the correlated calculations are made self-consistent by imposing the aforementioned matching conditions on the one-electron density matrices using the algorithm described in Sec. S4 - S6. Expectation values such as total energy are computed as a sum of local contributions (see Sec. S7). A global chemical potential is determined in both methods to fix the total number of electrons.

In all cases tested, the convergence of BE correlation energy with fragment size ( $n$ atoms) can be fitted to an exponential form,

$$
E_{\mathrm{BE}}^{\mathrm{corr}}=a b^{-n}+E_{\mathrm{BE}}^{0},
$$

for some constants $a, b$, and $E_{\mathrm{BE}}^{0}$, while the DMET0 results (if available) can be fitted to 
a surface-error decay,

$$
E_{\text {DMET0 }}^{\mathrm{corr}}=c n^{-d}+E_{\text {DMET0 }}^{0},
$$

for some other constants $c, d$, and $E_{\text {DMET0 }}^{0}$. We will perform extrapolation using these formulae to estimate the full-system embedding results.

To begin with, we study some simple 1D models where comparison to DMET is feasible. We first consider the hydrogen chain model, $\left(\mathrm{H}_{2}\right)_{m}$, where $m \mathrm{H}_{2}$ molecules form a chain with equal spacing between molecules $\left(d_{\mathrm{H}-\mathrm{H}}=0.75 \AA\right.$ and $\left.d_{\mathrm{H} \cdots \mathrm{H}}=1.05 d_{\mathrm{H}-\mathrm{H}}\right)$. This system is ideal for demonstrating the convergence behaviors of $\mathrm{BE}$ as discussed above. In a minimal basis of one orbital per hydrogen, the system is half-filled (i.e., $N_{\text {occ }} / N=1 / 2$ ) and hence the embedding stays in regime 1 . As we increase the basis size - which decreases the electron filling - the embedding will begin in regime 1 for small fragments, but transition into regime 2 for larger fragments.

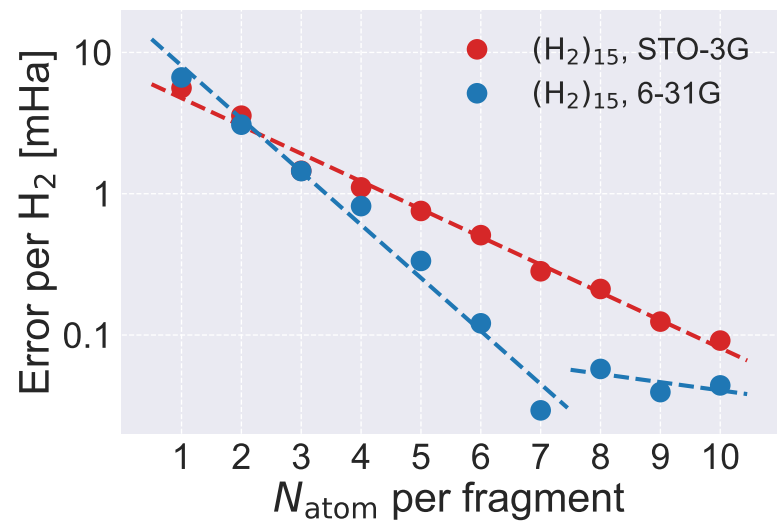

Figure 2: Variation of absolute error of BE energy $\left(\right.$ per $\left.\mathrm{H}_{2}\right)$ with fragment size for $\left(\mathrm{H}_{2}\right)_{15}$ in STO-3G (red) and 6-31G (blue) bases. Dashed curves are exponential fit.

The results for $\left(\mathrm{H}_{2}\right)_{15}$ using a minimal (STO$\left.3 \mathrm{G}^{45}\right)$ and a double-zeta $\left(6-31 \mathrm{G}^{46}\right)$ bases are presented in Figure 2. The decay of BE energy error in STO-3G is well-fitted to a single exponential function, confirming the embedding staying in regime 1 . For $6-31 \mathrm{G}$, however, two distinct regimes of exponential convergence are observed, with the error decaying much faster in regime 1 . The transition occurs between $n=7$ and 8 , which is exactly when $N_{f}$ starts exceed- ing $N_{\text {occ. }}$. In both cases, the extrapolated total energy using the data in regime 1 achieves chemical accuracy (Table 1), highlighting the efficacy of recovering valence electron correlation. By contrast, the convergence of DMET0 energy is much noisier (Figure S1) and cannot be effectively extrapolated.

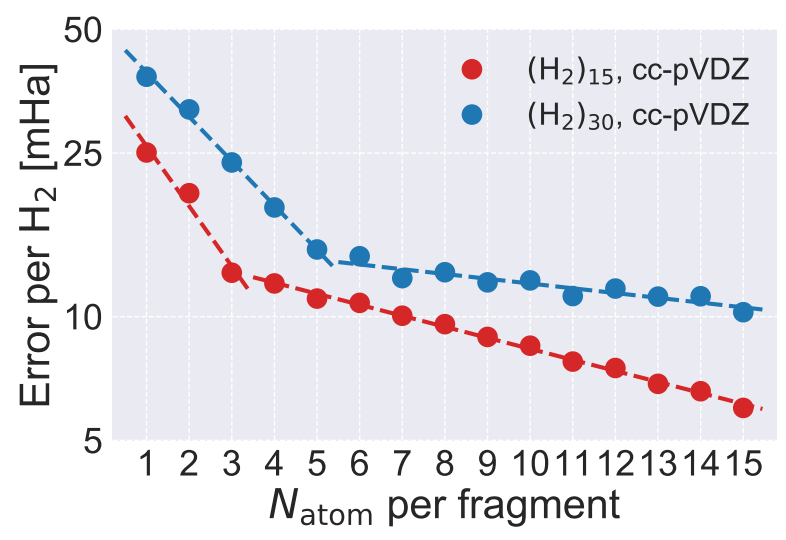

Figure 3: Variation of absolute error of BE energy $\left(\right.$ per $\mathrm{H}_{2}$ ) with fragment size for $\left(\mathrm{H}_{2}\right)_{15}$ (red) and $\left(\mathrm{H}_{2}\right)_{30}$ (blue) in cc-pVDZ basis. Dashed curves are exponential fit.

To further illustrate the convergence behavior, we perform calculations for $\left(\mathrm{H}_{2}\right)_{15}$ and $\left(\mathrm{H}_{2}\right)_{30}$ using a larger basis, cc-pVDZ, ${ }^{47}$ where each hydrogen is described by five orbitals (two $1 s$ 's and three $2 p$ 's). The results are shown in Figure 3. As in the 6-31G basis, a fast exponential decay followed by a slower one is observed here, but the transition between the two regimes occurs much earlier due to the further reduced electron filling $\left(N_{\text {occ }} / N=1 / 10\right)$. Unlike in small bases, however, the early arrival of regime 2 results in a relatively large error even using fragments of 15 atoms. Furthermore, comparison of $\left(\mathrm{H}_{2}\right)_{15}$ and $\left(\mathrm{H}_{2}\right)_{30}$ demonstrates that when SD-based embedding is applied to larger and larger systems, the transition from regime 1 to regime 2 comes later and later. Thus, in a large molecule with a large basis set, only the regime 1 behavior is likely to be accessed. Unfortunately, as detailed in the Supporting Information, extrapolation based on the data in regime 1 is not accurate for $\left(\mathrm{H}_{2}\right)_{30}$, leading to errors on the order of tens of $\mathrm{kcal} / \mathrm{mol}$ both for BE and DMET0 (See Figure S7 and Table S1). We thus conclude that, 
as presently constructed, SD-based embedding methods with mean field baths are only wellsuited to recovering the valence correlation in molecular systems.

We should note that, in addition to the intrinsic inaccuracies addressed above, which are quite general, there are some additional limitations imposed by the artificial $\left(\mathrm{H}_{2}\right)_{m}$ chain examples. First, in addition to the intrinsic disentanglement in regime 2 , the $2 p$ orbitals perpendicular to the molecular chain also lead to accidental disentanglement even in regime $1 .{ }^{16}$ Second, we find that the AOs on a given atom are not very effective at capturing entanglement with distant atoms (e.g. those separated by several chemical bonds) leading to additional linear dependence issues that get worse as $m$ increases. The result is that the error per $\mathrm{H}_{2}$ in $\left(\mathrm{H}_{2}\right)_{30}$ is significantly larger than in $\left(\mathrm{H}_{2}\right)_{15}$ for a given fragment size. For well-behaved cases without linear dependence, we do not generally observe this behavior. In the polyacetylene and polyacene cases discussed below, for example, for a given fragment size the error per atom is essentially independent of molecular size (Figure S3 and Table 1).

Table 1: Error of extrapolated total correlation energy (units: $\mathrm{kcal} / \mathrm{mol}$ ) of BE and DMET0 based on a series of small-fragment calculations for several molecules. MP2 correlation energy (units: Ha) of the full-system is included for reference. The extrapolation procedure is described in Supporting Information. A full list of fitted parameters can be found in Table S1.

\begin{tabular}{cccccc}
\hline \multirow{2}{*}{ Molecule } & $\mathrm{MP} 2$ & & $\mathrm{BE}$ & & $\mathrm{DMET0}$ \\
\cline { 2 - 3 } \cline { 5 - 5 } \cline { 5 - 5 } & $\mathrm{Ha}$ & $\mathrm{kcal} / \mathrm{mol}$ & & $\mathrm{kcal} / \mathrm{mol}$ \\
\hline$\left(\mathrm{H}_{2}\right)_{15}, \mathrm{SZ}$ & -0.28684 & & 0.35 & & $\mathrm{~N} / \mathrm{A}$ \\
$\left(\mathrm{H}_{2}\right)_{15}, \mathrm{DZ}$ & -0.38172 & & 0.41 & & $\mathrm{~N} / \mathrm{A}$ \\
$\mathrm{C}_{16} \mathrm{H}_{18}$ & -1.43273 & & -1.33 & & -11.05 \\
$\mathrm{C}_{20} \mathrm{H}_{22}$ & -1.79128 & & -1.65 & & -17.70 \\
Anthracene & -1.23285 & & 0.33 & & $\mathrm{~N} / \mathrm{A}$ \\
Tetracene & -1.58976 & & -0.90 & & $\mathrm{~N} / \mathrm{A}$ \\
Pentacene & -1.94788 & & -2.61 & & $\mathrm{~N} / \mathrm{A}$ \\
\hline
\end{tabular}

As a slightly more realistic example that can still be treated with DMET we study polyacetylenes, in $6-31 \mathrm{G}$ basis. ${ }^{48}$ The results are displayed in Figure S3. Overall, the convergence patterns observed above hold here, too. BE energy converges exponentially with fragment size, leading to chemical-accuracy extrapolation (Table 1). By contrast, DMET0 energy converges much more slowly and the extrapolated errors are an order of magnitude higher than BE. We emphasize that the BE matching conditions play a crucial role here, as is evident from the slow convergence of the one-shot BE energy (Figure S4). We also note that the aforementioned linear dependence issues are not significant in the small basis used here: calculations on 8-mer and 10-mer show similar accuracy.

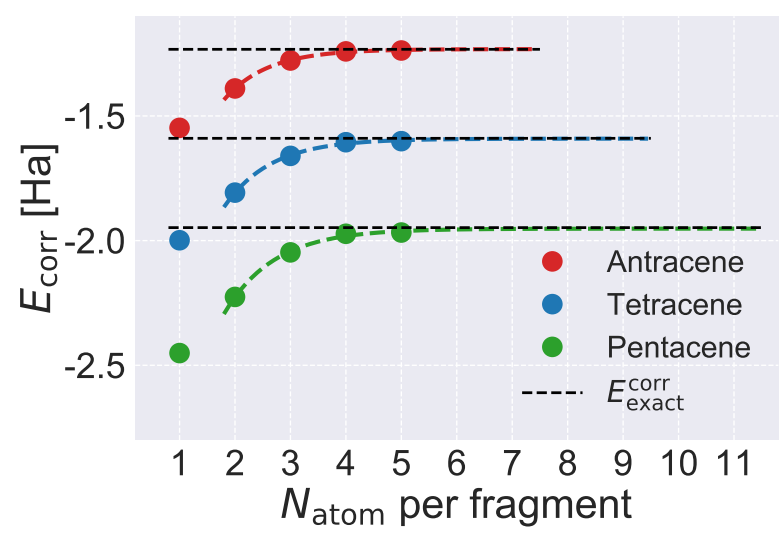

Figure 4: BE correlation energy (dots) and the exponential fit (dashed curves) according to eqn (6) for three polyacene molecules in 6-31G basis. Fragments of $2 \sim 5$ atoms are used in the fitting. The fitted parameters are listed in Table S1, and the extrapolated results in Talbe 1. $E_{\text {exact }}^{\text {corr }}$ denotes the full-system MP2 results.

The quasi-1D geometries used above are not a requirement by our atom-based fragment definition - they are chosen only to facilitate the comparison with DMET. For more complicated geometries, partitioning into non-overlapping fragments becomes ambiguous, and DMET (and other embedding methods) are often used in a regional manner, ${ }^{19,25,49}$ which works only if the chemistry of interest happens in a confined region. As a proof of concept, we study polyacenes in 6-31G basis, a system 
where all atomic groups are strongly coupled through $\pi$-conjugation. Figure 4 shows the correlation energy of BE using fragments containing up to five atoms. As in the quasi-1D systems, the exponential convergence of BE also holds here, leading to accurate extrapolation (Table 1).

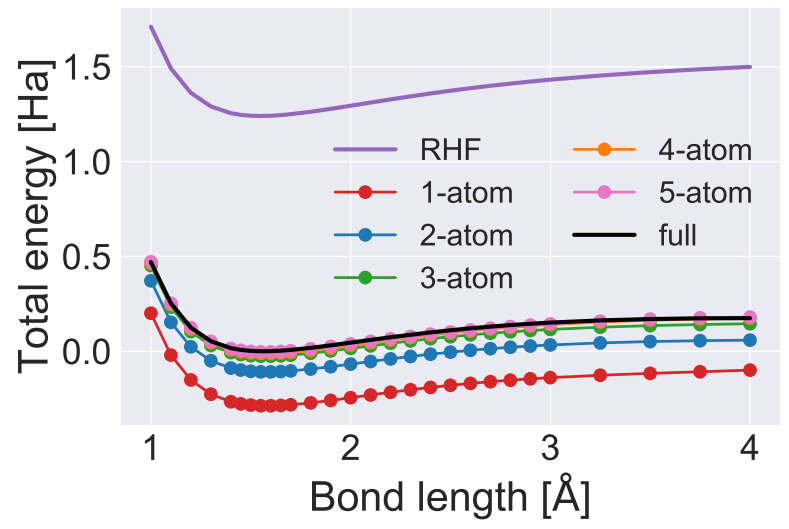

Figure 5: BE energy curves of the homolytic cleavage of diphenylethane computed using 6$31 \mathrm{G}$ basis and enlarging fragments up to five atoms. The fragments determined at equilibrium geometry is used for all bond lengths to avoid discontinuous change of energy. RHF (violet) and full-system MP2 (black) results are also included for comparison. The $y$-axis is shifted by the lowest point of the full-system MP2 curve.

In addition to molecules at equilibrium geometry, atom-based BE can handle bond dissociation as well. This is demonstrated in Figure 5 for the homolytic cleavage of diphenylethane, $\left(\mathrm{PhCH}_{2}\right)_{2} \longrightarrow 2 \mathrm{PhCH}_{2} \cdot$, using a 6-31G basis. A frequent problem of applying embedding methods to systems with varying geometries is that the energy curves may be discontinuous due to abrupt changes in fragment definition as the structure changes. To address this, we use the fragments determined at the equilibrium geometry for all calculations. As can be seen, smooth energy curves are obtained for all fragment sizes. Moreover, these curves converge quickly and monotonically to the full-system results at an exponential rate (Figure S5), even in the dissociated regime.

To summarize, we have presented Bootstrap Embedding with an atom-based fragment defi- nition that enables robust and black-box treatment of arbitrary molecular geometries. Numerical tests on various molecules suggest that with a mean-field bath, atom-based BE shows an exponential rate of convergence with fragment size, and is efficient at recovering valance electron correlation using extrapolation.

In the future, atom-based BE can be extended in several directions. First, the slow convergence of dynamic correlation energy in large bases (due to intrinsic and/or accidental disentanglement) could potentially be accelerated by either augmenting the entangled bath with virtual orbitals or adopting a better bath. ${ }^{22,23,32,33}$ Second, we can explore different local solvers including configuration interaction, ${ }^{2}$ coupledcluster, ${ }^{3}$ and multi-reference ${ }^{50-52}$ methods that might fit better to specific problems, as well as mean-field baths for excited states. ${ }^{53-56}$ Last but not least, it will be interesting to develop efficient implementation for handling systems of large size. With these potential future developments, we anticipate that atom-based $\mathrm{BE}$ may become a low-scaling and highly accurate approach to electron correlation in large molecules.

Acknowledgement HY thanks Henry K. Tran and Dr. Nathan D. Ricke for proofreading the manuscript. This work was funded by a grant from the NSF (Grant No. CHE-1464804). TV is a David and Lucille Packard Foundation Fellow.

Supporting Information (i) Supplementary figures (Sec. S1); (ii) details of extrapolation based on calculations using small fragments (Sec. S2); (iii) more formal and detailed discussion on BE (Secs. S3 - S8); (iv) geometries of all molecules investigated in this work (Sec. S9).

\section{References}

(1) Møller, C.; Plesset, M. S. Note on an Approximation Treatment for Many-Electron Systems. Phys. Rev. 1934, 46, 618-622.

(2) Sherrill, C. D.; Schaefer, H. F. In The Configuration Interaction Method: Ad- 
vances in Highly Correlated Approaches; Löwdin, P.-O., Sabin, J. R., Zerner, M. C., Brändas, E., Eds.; Adv. Quantum Chem.; Academic Press, 1999; Vol. 34; pp 143 269.

(3) Bartlett, R. J.; Musiał, M. Coupledcluster theory in quantum chemistry. Rev. Mod. Phys. 2007, 79, 291-352.

(4) Olivares-Amaya, R.; $\mathrm{Hu}, \mathrm{W}$; Nakatani, N.; Sharma, S.; Yang, J.; Chan, G. K.-L. The ab-initio density matrix renormalization group in practice. J. Chem. Phys. 2015, 142, 034102.

(5) Hohenberg, P.; Kohn, W. Inhomogeneous Electron Gas. Phys. Rev. 1964, 136, B864-B871.

(6) Kohn, W.; Sham, L. J. Self-Consistent Equations Including Exchange and Correlation Effects. Phys. Rev. 1965, 140, A1133-A1138.

(7) Cohen, A. J.; Mori-Sánchez, P.; Yang, W. Insights into Current Limitations of Density Functional Theory. Science 2008, 321, 792-794.

(8) Cohen, A. J.; Mori-Sánchez, P.; Yang, W. Challenges for Density Functional Theory. Chem. Rev. 2012, 112, 289-320, PMID: 22191548.

(9) Medvedev, M. G.; Bushmarinov, I. S.; Sun, J.; Perdew, J. P.; Lyssenko, K. A. Density functional theory is straying from the path toward the exact functional. Science 2017, 355, 49-52.

(10) Huang, C.; Carter, E. A. Potentialfunctional embedding theory for molecules and materials. J. Chem. Phys. 2011, 135, 194104

(11) Manby, F. R.; Stella, M.; Goodpaster, J. D.; Miller, T. F. A Simple, Exact Density-Functional-Theory Embedding Scheme. J. Chem. Theory Comput. 2012, 8, 2564-2568.
(12) Goodpaster, J. D.; Barnes, T. A.; Manby, F. R.; Miller, T. F. Accurate and systematically improvable density functional theory embedding for correlated wavefunctions. J. Chem. Phys. 2014, 140, 18A507.

(13) Knizia, G.; Chan, G. K.-L. Density Matrix Embedding: A Simple Alternative to Dynamical Mean-Field Theory. Phys. Rev. Lett. 2012, 109, 186404.

(14) Knizia, G.; Chan, G. K.-L. Density Matrix Embedding: A Strong-Coupling Quantum Embedding Theory. J. Chem. Theory Comput. 2013, 9, 1428-1432.

(15) Welborn, M.; Tsuchimochi, T.; Van Voorhis, T. Bootstrap embedding: An internally consistent fragment-based method. J. Chem. Phys. 2016, 145, 074102 .

(16) Ye, H.-Z.; Welborn, M.; Ricke, N. D.; Van Voorhis, T. Incremental embedding: A density matrix embedding scheme for molecules. J. Chem. Phys. 2018, 149, 194108.

(17) Lan, T. N.; Kananenka, A. A.; Zgid, D. Communication: Towards ab initio selfenergy embedding theory in quantum chemistry. J. Chem. Phys. 2015, 143, 241102.

(18) Lan, T. N.; Zgid, D. Generalized SelfEnergy Embedding Theory. J. Phys. Chem. Lett. 2017, 8, 2200-2205.

(19) Yu, K.; Carter, E. A. Extending density functional embedding theory for covalently bonded systems. Proc. Natl. Acad. Sci. 2017, 114, E10861-E10870.

(20) Peschel, I.; Eisler, V. Reduced density matrices and entanglement entropy in free lattice models. J. Phys. A: Math. Theor. 2009, 42, 504003.

(21) Peschel, I. Special Review: Entanglement in Solvable Many-Particle Models. Braz. J. Phys. 2012, 4R, 267-291. 
(22) Bulik, I. W.; Scuseria, G. E.; Dukelsky, J. Density matrix embedding from broken symmetry lattice mean fields. Phys. Rev. B 2014, 89, 035140 .

(23) Zheng, B.-X.; Chan, G. K.-L. Groundstate phase diagram of the square lattice Hubbard model from density matrix embedding theory. Phys. Rev. B 2016, 93, 035126 .

(24) Bulik, I. W.; Chen, W.; Scuseria, G. E. Electron correlation in solids via density embedding theory. J. Chem. Phys. 2014, $141,054113$.

(25) Wouters, S.; Jiménez-Hoyos, C. A.; Sun, Q.; Chan, G. K.-L. A Practical Guide to Density Matrix Embedding Theory in Quantum Chemistry. J. Chem. Theory Comput. 2016, 12, 2706-2719, PMID: 27159268.

(26) Pham, H. Q.; Bernales, V.; Gagliardi, L. Can Density Matrix Embedding Theory with the Complete Activate Space SelfConsistent Field Solver Describe Single and Double Bond Breaking in Molecular Systems? J. Chem. Theory Comput. 2018, 14, 1960-1968.

(27) Tran, H. K.; Van Voorhis, T.; Thom, A. J. W. Using SCF metadynamics to extend density matrix embedding theory to excited states. J. Chem. Phys. 2019, 151, 034112 .

(28) Maier, T.; Jarrell, M.; Pruschke, T.; Hettler, M. H. Quantum cluster theories. Rev. Mod. Phys. 2005, 77, 1027-1080.

(29) Koch, E.; Sangiovanni, G.; Gunnarsson, O. Sum rules and bath parametrization for quantum cluster theories. Phys. Rev. B 2008, 78, 115102.

(30) Ricke, N.; Welborn, M.; Ye, H.-Z.; Van Voorhis, T. Performance of Bootstrap Embedding for long-range interactions and 2D systems. Mol. Phys. 2017, 115, 22422253 .
(31) Ye, H.-Z.; Ricke, N. D.; Tran, H. K.; Van Voorhis, T. Bootstrap Embedding for Molecules. J. Chem. Theory Comput. 2019, 15, 4497-4506.

(32) Tsuchimochi, T.; Welborn, M.; Van Voorhis, T. Density matrix embedding in an antisymmetrized geminal power bath. J. Chem. Phys. 2015, 143, 024107.

(33) Hermes, M. R.; Gagliardi, L. Multiconfigurational Self-Consistent Field Theory with Density Matrix Embedding: The Localized Active Space Self-Consistent Field Method. J. Chem. Theory Comput. 2019, 15, 972-986.

(34) Fertitta, E.; Booth, G. H. Rigorous wave function embedding with dynamical fluctuations. Phys. Rev. B 2018, 98, 235132.

(35) Fertitta, E.; Booth, G. H. Energyweighted density matrix embedding of open correlated chemical fragments. $J$. Chem. Phys. 2019, 151, 014115.

(36) Huang, C. Embedded Cluster Density Approximation for ExchangeâĂŞCorrelation Energy: A Natural Extension of the Local Density Approximation. J. Chem. Theory Comput. 2018, 14, 6211-6225.

(37) Chen, M.; Baer, R.; Neuhauser, D.; Rabani, E. Overlapped embedded fragment stochastic density functional theory for covalently-bonded materials. J. Chem. Phys. 2019, 150, 034106.

(38) Nobes, R. H.; Moncrieff, D.; Wong, M. W.; Radom, L.; Gill, P. M.; Pople, J. A. The structure and stability of the $\mathrm{O}_{2}^{+2}$ dication: a dramatic failure of Møller-Plesset perturbation theory. Chem. Phys. Lett. 1991, 182, $216-224$.

(39) Soydaş, E.; Bozkaya, U. Assessment of Orbital-Optimized MP2.5 for Thermochemistry and Kinetics: Dramatic Failures of Standard Perturbation Theory Approaches for Aromatic Bond Dissociation 
Energies and Barrier Heights of Radical Reactions. J. Chem. Theory Comput. 2015, 11, 1564-1573.

(40) Boys, S. F. Construction of Some Molecular Orbitals to Be Approximately Invariant for Changes from One Molecule to Another. Rev. Mod. Phys. 1960, 32, 296-299.

(41) Shao, Y.; Gan, Z.; Epifanovsky, E.; Gilbert, A. T.; Wormit, M.; Kussmann, J.; Lange, A. W.; Behn, A.; Deng, J.; Feng, X.; Ghosh, D.; Goldey, M.; Horn, P. R.; Jacobson, L. D.; Kaliman, I.; Khaliullin, R. Z.; Kuś, T.; Landau, A.; Liu, J.; Proynov, E. I.; Rhee, Y. M.; Richard, R. M.; Rohrdanz, M. A.; Steele, R. P.; Sundstrom, E. J.; III, H. L. W.; Zimmerman, P. M.; Zuev, D.; Albrecht, B.; Alguire, E.; Austin, B.; Beran, G. J. O.; Bernard, Y. A.; Berquist, E.; Brandhorst, K.; Bravaya, K. B.; Brown, S. T.; Casanova, D.; Chang, C.M.; Chen, Y.; Chien, S. H.; Closser, K. D.; Crittenden, D. L.; Diedenhofen, M.; Jr., R. A. D.; Do, H.; Dutoi, A. D.; Edgar, R. G.; Fatehi, S.; Fusti-Molnar, L.; Ghysels, A.; Golubeva-Zadorozhnaya, A.; Gomes, J.; Hanson-Heine, M. W.; Harbach, P. H.; Hauser, A. W.; Hohenstein, E. G.; Holden, Z. C.; Jagau, T.-C.; Ji, H.; Kaduk, B.; Khistyaev, K.; Kim, J.; Kim, J.; King, R. A.; Klunzinger, P.; Kosenkov, D.; Kowalczyk, T.; Krauter, C. M.; Lao, K. U.; Laurent, A. D.; Lawler, K. V.; Levchenko, S. V.; Lin, C. Y.; Liu, F.; Livshits, E.; Lochan, R. C.; Luenser, A.; Manohar, P.; Manzer, S. F.; Mao, S.P.; Mardirossian, N.; Marenich, A. V.; Maurer, S. A.; Mayhall, N. J.; Neuscamman, E.; Oana, C. M.; OlivaresAmaya, R.; O’Neill, D. P.; Parkhill, J. A.; Perrine, T. M.; Peverati, R.; Prociuk, A.; Rehn, D. R.; Rosta, E.; Russ, N. J.; Sharada, S. M.; Sharma, S.; Small, D. W.; Sodt, A.; Stein, T.; Stück, D.; Su, Y.C.; Thom, A. J.; Tsuchimochi, T.; Vanovschi, V.; Vogt, L.; Vydrov, O.; Wang, T.; Watson, M. A.; Wenzel, J.;
White, A.; Williams, C. F.; Yang, J.; Yeganeh, S.; Yost, S. R.; You, Z.-Q.; Zhang, I. Y.; Zhang, X.; Zhao, Y.; Brooks, B. R.; Chan, G. K.; Chipman, D. M.; Cramer, C. J.; III, W. A. G.; Gordon, M. S.; Hehre, W. J.; Klamt, A.; III, H. F. S.; Schmidt, M. W.; Sherrill, C. D.; Truhlar, D. G.; Warshel, A.; $\mathrm{Xu}$, X.; Aspuru-Guzik, A.; Baer, R.; Bell, A. T.; Besley, N. A.; Chai, J.-D.; Dreuw, A.; Dunietz, B. D.; Furlani, T. R.; Gwaltney, S. R.; Hsu, C.-P.; Jung, Y.; Kong, J.; Lambrecht, D. S.; Liang, W.; Ochsenfeld, C.; Rassolov, V. A.; Slipchenko, L. V.; Subotnik, J. E.; Van Voorhis, T.; Herbert, J. M.; Krylov, A. I.; Gill, P. M.; Head-Gordon, M. Advances in molecular quantum chemistry contained in the Q-Chem 4 program package. Mol. Phys. 2015, 113, 184-215.

(42) Ye, H.-Z. Frankenstein: An Electronic Structure Method Development Platform. https://github.com/hongzhouye/ frankenstein, 2019.

(43) Valeev, E. F. Libint: A library for the evaluation of molecular integrals of manybody operators over Gaussian functions. http://libint.valeyev.net, 2018.

(44) Sun, Q.; Berkelbach, T. C.; Blunt, N. S.; Booth, G. H.; Guo, S.; Li, Z.; Liu, J.; McClain, J. D.; Sayfutyarova, E. R.; Sharma, S.; Wouters, S.; Chan, G. K. PySCF: the Python-based simulations of chemistry framework. 2017; https://onlinelibrary.wiley.com/ doi/abs/10.1002/wcms. 1340.

(45) Hehre, W. J.; Stewart, R. F.; Pople, J. A. Self-Consistent Molecular-Orbital Methods. I. Use of Gaussian Expansions of Slater-Type Atomic Orbitals. J. Chem. Phys. 1969, 51, 2657-2664.

(46) Ditchfield, R.; Hehre, W. J.; Pople, J. A. Self-Consistent Molecular-Orbital Methods. IX. An Extended Gaussian-Type Basis for Molecular-Orbital Studies of Or- 
ganic Molecules. J. Chem. Phys. 1971, 54, 724-728.

(47) Jr., T. H. D. Gaussian basis sets for use in correlated molecular calculations. I. The atoms boron through neon and hydrogen. J. Chem. Phys. 1989, 90, 1007-1023.

(48) Hehre, W. J.; Ditchfield, R.; Pople, J. A. Self-Consistent Molecular Orbital Methods. XII. Further Extensions of GaussianType Basis Sets for Use in Molecular Orbital Studies of Organic Molecules. J. Chem. Phys. 1972, 56, 2257-2261.

(49) Zhang, X.; Carter, E. A. Subspace Density Matrix Functional Embedding Theory: Theory, Implementation, and Applications to Molecular Systems. J. Chem. Theory Comput. 2019, 15, 949-960.

(50) Siegbahn, P.; Heiberg, A.; Roos, B.; Levy, B. A Comparison of the SuperCI and the Newton-Raphson Scheme in the Complete Active Space SCF Method. Phys. Scr. 1980, 21, 323-327.

(51) Roos, B. O.; Taylor, P. R.; Siegbahn, P. E. A complete active space SCF method (CASSCF) using a density matrix formulated super-CI approach. Chem. Phys. 1980, 48, $157-173$.

(52) Siegbahn, P. E. M.; Almlöf, J.; Heiberg, A.; Roos, B. O. The complete active space SCF (CASSCF) method in a Newton-Raphson formulation with application to the HNO molecule. $J$. Chem. Phys. 1981, 74, 2384-2396.

(53) Gilbert, A. T. B.; Besley, N. A.; Gill, P. M. W. Self-Consistent Field Calculations of Excited States Using the Maximum Overlap Method (MOM). J. Phys. Chem. A 2008, 112, 13164 - 13171.

(54) Thom, A. J. W.; Head-Gordon, M. Locating Multiple Self-Consistent Field Solutions: An Approach Inspired by Metadynamics. Phys. Rev. Lett. 2008, 101, 193001.
(55) Thom, A. J. W.; Head-Gordon, M. Hartree-Fock solutions as a quasidiabatic basis for nonorthogonal configuration interaction. J. Chem. Phys. 2009, 131, 124113.

(56) Ye, H.-Z.; Welborn, M.; Ricke, N. D.; Van Voorhis, T. $\sigma$-SCF: A direct energytargeting method to mean-field excited states. J. Chem. Phys. 2017, 147, 214104. 